\title{
Towards Washable Wearable Antennas: A Comparison of Coating Materials for Screen-Printed Textile-Based UHF RFID Tags
}

\author{
Tiiti Kellomäki, ${ }^{1}$ Johanna Virkki, ${ }^{1}$ Sari Merilampi, ${ }^{2}$ and Leena Ukkonen ${ }^{3}$ \\ ${ }^{1}$ Department of Electronics, Tampere University of Technology, P.O. Box 692, 33101 Tampere, Finland \\ ${ }^{2}$ Faculty of Technology and Maritime Management, Satakunta University of Applied Sciences, Tekniikantie 2, 28600 Pori, Finland \\ ${ }^{3}$ Rauma Research Unit, Department of Electronics, Tampere University of Technology, 26100 Rauma, Finland
}

Correspondence should be addressed to Tiiti Kellomäki, tiiti.kellomaki@tut.fi

Received 10 August 2012; Accepted 14 September 2012

Academic Editor: Panayiotis Petratos

Copyright () 2012 Tiiti Kellomäki et al. This is an open access article distributed under the Creative Commons Attribution License, which permits unrestricted use, distribution, and reproduction in any medium, provided the original work is properly cited.

\begin{abstract}
(Radio frequency identification) RFID tags integrated into clothing enable monitoring of people without their conscious effort. This requires tags to be an unnoticeable part of clothing and comfortable to wear. In this study, RFID antennas were screen printed on two different fabrics, six different coating materials for the (integrated circuits) ICs were applied, and the reliability of these RFID tags was tested with moisture and laundry tests. Generally, glue-type coating materials were easier to handle and could be spread precisely. All the tags were operational immediately after the coatings were applied, and five of the coating materials were seen to protect the IC from detaching in the laundry. It was found that the uneven fabric surface caused discontinuities and breaks in narrow conductors, and thus hard coatings may also be needed to keep the tag from breaking in laundry.
\end{abstract}

\section{Introduction}

The emerge of body-centric wireless communications and the potential and possibilities of wearable healthcare and monitoring systems have created a need for fabric-based antennas which could be seamlessly integrated with clothing. With body-worn antennas it is possible to hide the technology that often frightens patients, while discreetly providing assistance. Unnoticed monitoring instruments are needed in involuntary treatment and dementia care, where the patients may seek to remove suspicious devices.

In welfare industry, there is a growing need for on-body sensing with unnoticeable and comfortable sensor components, such as wearable sensors. For example, the lack of personnel in nursing homes is a significant problem. Instead of replacing human contact, technology should be assistive and take care of certain monitoring tasks, thus saving the time of the professional staff for more important tasks. The current trend is to help the elderly live at home instead of caring homes. To facilitate this, technology is needed to improve the feeling of security, for example. Sensing of breathing, regular actions (such as bathroom visits), body temperature, sleep, or body limb movement can help in automatic monitoring of well-being. In rehabilitation process, sensors could monitor that the prescribed exercises are performed but not too hard training is done.

The first step in utilizing RFID (radio frequency identification) in healthcare is to monitor, track, and identify patients who carry tags in their wristbands. Presently, patients are often identified using a barcode wristband at bedside, before giving away drugs. Because RFID tags require no line-of-sight, they can be read, for example, through bed sheets without disturbing the sleeping patient. With read ranges of several metres, UHF (ultrahigh frequency) tags enable monitoring of people without their conscious effort.

Instead of just tracking people, RFID tags offer more possibilities. RFID ICs (integrated circuits) with integrated sensors are available, such as in [1] with a temperature sensor and a possibility of connecting external analogue sensors. In such a system the tag is responsible for the communications link from the patient to the reader, while the sensory data is provided by external sensors. Readers can be embedded, for example, in the hospital bed or even the mobile phone of the user. Different data can be queried at predefined intervals and transmitted to a hospital database for automatic processing, or only on-demand for the benefit of the user. 
Printing methods can be used to fabricate wearable UHF RFID tag antennas easily, cost effectively, and reliably. Screen printing is a versatile technique, which is already used in the electronics industry to print thick film structures like conductors, dielectrics and passive components, and membrane touch switches, and also to print coatings such as solder-masks and resists. The technique is thus familiar to electronics manufacturers $[2,3]$ and naturally also to textile industry.

Wearable UHF RFID tags need to be able to stand different environmental conditions and continuous washing. This requires the antenna to be washable and the IC to be protected. In this study, RFID tag antennas were screen printed on two different fabrics, six different coating materials for the ICs were applied, and the reliability of these RFID tags was tested with moisture and laundry tests.

The next section gives a short review of RFID and wearable antennas. Section 3 introduces the tag under test, as well as the fabrication method. The coating materials and reliability tests are summarized in Section 4. Finally, Section 5 discusses the results: effect of fabric material and coating, and the outcomes of the soaking and laundry tests.

\section{Wearable RFID Tags}

An RFID system consists of a reader and tags. When the reader transmits a query, the tags will respond, for example, by sending their individual code. In a passive system, the tags contain no internal power source, but are powered up by the energy transmitted by the reader, and the response link is based on backscattering modulation. A passive RFID tag consists of an antenna and an IC chip that contains all the required electronics. For optimal power transfer and performance, the antenna needs to be conjugate matched to the IC impedance, in our case (Alien Higgs-2 microchip) $17+j 137 \mathrm{ohms}$ at the centre frequency of $866 \mathrm{MHz}$ [4].

RFID tags are already widely used in access control in the form of keycards that operate at $13.56 \mathrm{MHz}$ and can be read from a distance of few millimetres. Clip-on UHF tags are used in hospitals to provide an alert when a patient with a memory disorder attempts to walk out from the front door, but usually only one or two doors are capable of sensing tags. In a more complete system, every door could include an RFID reader and open only to those with permission. Such tracking systems are also useful in old peoples' homes and other public institutions such as nursing homes for mentally disabled people. This requires comfortable and unobtrusive tags, and in the case of escaping patients, tags that are difficult to notice and to remove. One solution is to integrate the tag into clothing. Prototypes of wearable tags are reviewed in [5]. Tags that are part of clothing could also be used in tracking laundry in institutions to avoid clothes to be lost through the laundry service.

The advantages of screen-printed textile antennas include that the screen printing is a fast and "easy," relatively inexpensive method that can also be used in mass-production. Relatively high resolution and flexible pattern are also achieved on fabric. On smooth substrates, the resolution is approximately $50 \mu \mathrm{m}$ line width (industrial production).
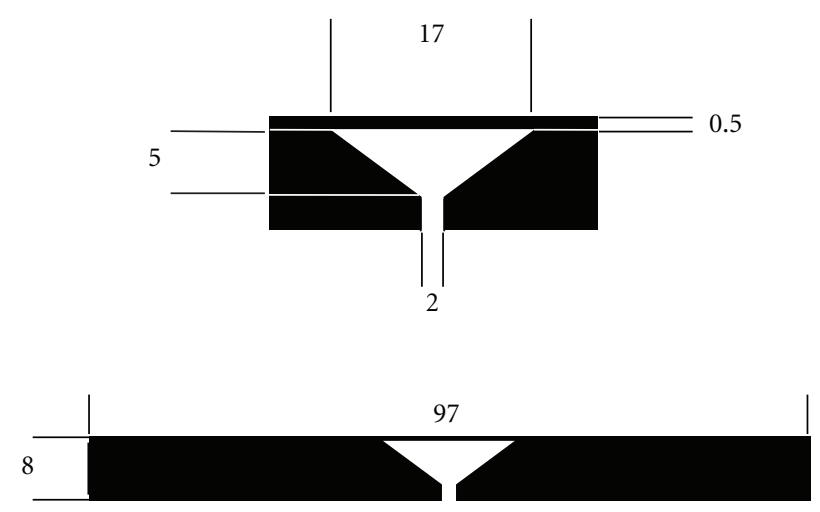

FIGURE 1: The geometry of the screen-printed tags. Dimensions are in $\mathrm{mm}$.

However, on uneven absorbent materials, the resolution is more limited. For example, according to [6], a minimum line resolution of $200 \mu \mathrm{m}$ can be achieved on $100 \mu \mathrm{m}$ thick polyester fabric (thickness of the film wire is $10 \mu \mathrm{m}$ ). However, the resolution depends on the roughness of surface: structure of the fabric, mesh of the fabric, thickness of the yarns, and so forth.

In addition to screen printing [7-9], wearable antennas have been fabricated of conducting textiles $[10,11]$, conductive ribbons [12], and copper meshes [13], embroidered using conductive thread $[12,14]$ and sprayed using conductive paint [12]. Copper tape is often used for first prototypes, but it easily breaks in use and cannot be used in consumer products. In articles [15-19], conducting threads or yarns were used for interconnection of electronic devices with fabrics. In case of using conductive yarns, only perpendicular wiring structures can be used due to the intrinsic characteristics of fabric weaving.

There has been little research on the durability and washability of textile antennas. Zhu and Langley washed an antenna made of conductive textile and reported that its performance was unchanged after drying properly and that even a damp antenna was usable [11]. The effect of protective coating on antennas was studied in [20] where it was concluded that the effect of coating materials on antenna performance is far less significant than manufacturing tolerances. In this paper, we are concentrating on different coating materials and their ability to protect the IC and IC joint against washing. The printed antenna itself is left uncoated.

\section{Preparation of Wearable Tags by Screen Printing}

A dipole-type RFID tag geometry originally presented in [21] was used as the sample tag antenna in this study due to its simple structure and sufficient performance in previous studies on different substrates. The tag geometry is illustrated in Figure 1 [21].

The sample tags were manufactured by screen printing polymer thick film ink on fabric substrates. In screen printing, the ink is pressed through a screen, a fabric mesh of threads, onto the substrate with a squeegee (blade). 


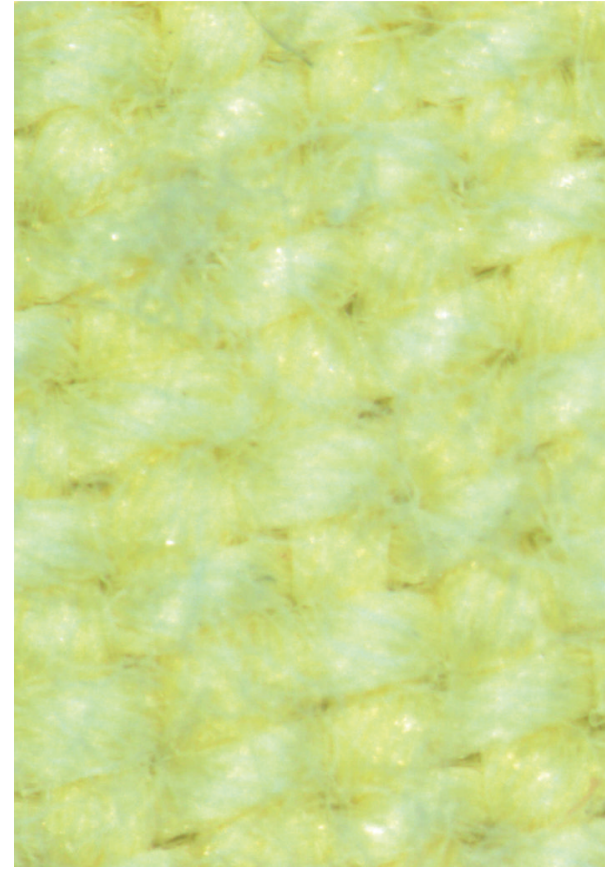

(a)

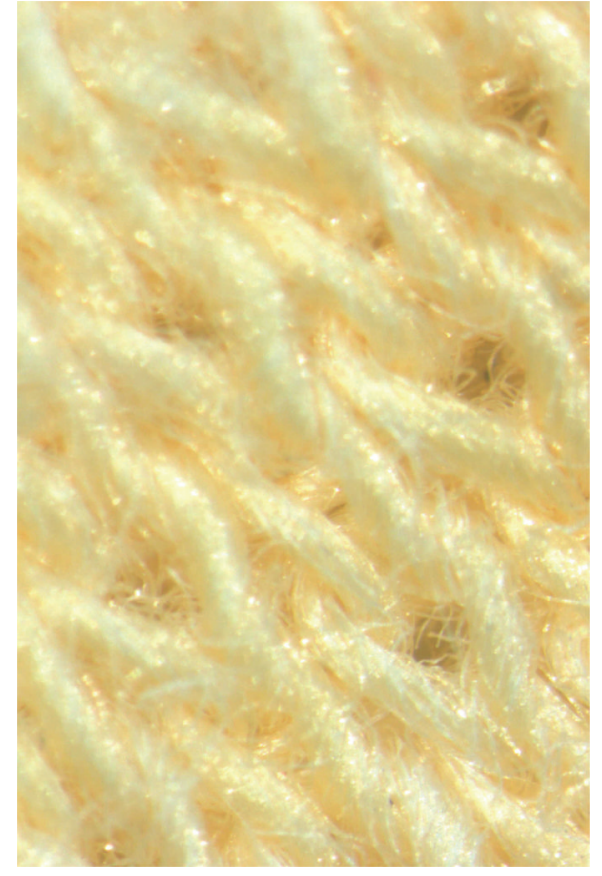

(b)

Figure 2: Fabrics (A) and (B), magnification is $\times 20$. Fabric (A) has gauge 30 threads/cm in warp, 20 threads/cm in weft; Fabric (B) has 13 stitches $/ \mathrm{cm}$ and 14 rows $/ \mathrm{cm}$.

Nonimage areas of the screen mesh are blocked out with a stencil, often an emulsion, and in the image areas the screen is left open.

Advantages of screen printing include low cost of equipment and ability to deposit thick films and large areas in one pass. There is also no need for pretreatment on uneven substrates such as fabrics. In passive UHF RFID tag manufacturing, the resolution is very seldom a limiting factor when selecting the printing process. Printed film thickness, however, is a critical parameter since it affects the electrical performance of the film.

In this study, flatbed screen printing was used in sample preparation. To provide a consistent and thick enough ink film, three cascade print cycles were performed. After printing, the samples were cured at $120^{\circ} \mathrm{C}$ for 15 minutes. If large batch sizes are to be printed, rotary-screen printing is the most suitable screen printing process, because it can be used in reel-to-reel manufacturing lines [22].

3.1. Screen Printable Ink. In screen printing, the ink is electrically conductive polymer thick film (PTF) material. PTF inks are polymer matrix composites. The PTF ink consists of metallic filler, binder material (polymer matrix), solvents, and additives. The ingredients, as well as their relative amounts, are selected according to the printing method. The filler of the ink is metal powder, commonly silver, which is also used in the structures discussed in this study. Typically, the particle size (the median of the particle size distribution) in screen printable silver inks is a few microns. The particles are usually in flake form and the size distribution is relatively large $[2,23,24]$.
The particles are suspended in vehicle for printing use. The vehicle normally consists of a nonvolatile and a volatile portion which will evaporate during curing. Solvents form the volatile part of the vehicle, and they are normally used to impart the desired characteristics of flow, viscosity, and ink density. The non-volatile portion of the vehicle is called binder. Binder is needed to hold the particles together once the ink is transferred onto a substrate, to affix them to the surface and to protect the film from being damaged. Binder materials in screen printing inks are resins. PTF inks also contain modifiers or additive ingredients, which are used to affect the characteristics of the ink. Characteristics of the screen printable ink, which was used in this study, are introduced in Table 1 [23-25].

3.2. Fabric Substrates. In this study, we wanted to form a general view of the performance of screen printed tags on fabric substrates. To fabricate samples where the ink films have different morphology and the substrates have different mechanical and absorbtion characteristics, two fabrics with different structures were selected for the sample tags (see Figure 2).

The first fabric (Fabric (A)) is a plain weave 100\% cotton fabric, typically used in bed sheets, for example. The second one (Fabric (B)) is a $35 \%$ cotton, $65 \%$ polyester interlock knitted fabric. This kind of fabric is typically used in clothes. Fabric (B) is very flexible while Fabric (A) is not. Crosssections of the fabricated antennas on Fabric (A) and (B) can be seen in Figure 3.

After printing and curing the tag antennas, Alien Higgs2 microchips [4] were attached to the antennas to form 
TABLE 1: Polymer thick film ink used in sample preparation.

\begin{tabular}{|c|c|c|c|}
\hline Manufacturer's description & Curing conditions & Viscosity & Conductivity \\
\hline $\begin{array}{l}\text { One component silver ink consisting of polyester resin and silver particles. Silver } \\
\text { content is } 60-65 \mathrm{wt} \% \text { and polyester resin content is } 11-14 \mathrm{wt} \% \text {. Particle sizes are } \\
\text { mainly in the range from } 3 \text { to } 15 \mu \mathrm{m}\end{array}$ & $120^{\circ} \mathrm{C}, 15 \mathrm{~min}$ & $20-30 \mathrm{~Pa} \cdot \mathrm{s}$ & $1.25 \mathrm{MS} / \mathrm{m}$ \\
\hline
\end{tabular}

TABLE 2: Coating materials used in this study.

\begin{tabular}{lccc}
\hline Name & Description & Product & Notes from manufacturer \\
\hline Acrylic & Acrylic & Buehler VariKleer [29] & \\
Epoxy & Epoxy & SpeciFix-20 [30] & Can be washed in $90^{\circ} \mathrm{C}$ \\
Latex & Natural rubber latex glue & Bison textile adhesive [31] & Withstands $+150^{\circ} \mathrm{C}$, water resistant \\
Silicone & Silicone elastomer & Casco silicone [32] & Withstands $+70^{\circ} \mathrm{C}$ \\
PVA1 & Polyvinyl acetate adhesive & Eri Keeper [33] & Withstands $+70^{\circ} \mathrm{C}$, water resistant \\
PVA2 & Water-resistant polyvinyl acetate adhesive & Eri Keeper Plus [33] & $(\mathrm{DIN}$ EN 204, class D3) \\
\hline
\end{tabular}

the tag. The chips were readily attached to larger pads by the chip manufacturer. This structure is called a strap. The straps were attached to the antennas using a conductive silver epoxy resin.

\section{Reliability and Performance Evaluation}

During field use, wearable RFID tags have to endure many kinds on environmental stresses, such as different temperatures, humidity, wear, and tear. One of the most significant stresses comes from the continuous laundry cycles. Thus, the antenna needs to be able to stand repetitive washing. In addition, in order to achieve reliable and durable tags against various environments, the shielding of the ICs needs to be considered. Six different coating materials (see Table 2) for ICs were chosen to be tested in this study: acrylic, epoxy, polyvinyl acetate adhesive (referred to as PVA1), waterresistant modified polyvinyl acetate adhesive (referred to as PVA2), silicone, and natural rubber latex glue (referred to as latex). For comparison purposes, we used tags with no coating on the IC.

The tags on two different textiles were evaluated in three reliability tests. Test 1 was a soaking test where tags with all the six coating materials were soaked. Test 2 was a laundry test where tags with all the six coating materials were washed once in a washing machine. Tests 1 and 2 aimed at characterising the coating material. Test 3 was a laundry test where two tag antennas (one antenna printed on Fabric (A) and one antenna printed on Fabric (B), both without coating on the IC) were washed in a washing machine for ten times, to see the effect of washing on the ink film itself. Liquid detergent without bleach was used in the laundry tests, but no softener was used. The washing cycle included washing in $60^{\circ} \mathrm{C}$, four rinses with cold water, and spinning with $1000 \mathrm{rpm}$, with a total duration of approximately 2.5 hours. The washing machine was filled with regular laundry, and the tags were placed in a lingerie bag. The tags were air-dried in room temperature after the moisture and laundry tests.
The performance of the tags was measured using the Tagformance Lite RFID measurement unit [26] in a small anechoic chamber. The measurement unit acts as a reader whose frequency and power are tunable. A frequency sweep was made, and at each frequency we recorded the lowest transmitter power to activate the tag. This is called the threshold power. Knowing the reader antenna gain, the measurement distance, and the sensitivity of the IC, we can calculate the read range and the realised gain of the tag antenna. The theoretical read range is calculated from $d=R \sqrt{\mathrm{EIRP} / P_{T}}$, where $R$ is the measurement distance, EIRP the maximal equivalent isotropic radiated power allowed by the regulations $3.28 \mathrm{~W}$ in Europe), and $P_{T}$ the threshold power of the tag under test, expressed as equivalent isotropic radiated power from the transmitter. Knowing that the sensitivity of the IC is $-14 \mathrm{dBm}$ [4], the realised gain (gain reduced by the losses due to impedance mismatch) of the tag is calculated by $G_{\text {real }}(\mathrm{dBi})=$ $-14 \mathrm{dBm}-10 \log \left(P_{T}(\lambda / 4 \pi R)^{2}\right)$, where $\lambda$ is the wavelength.

All measurements were made in copolarisation (vertical), with the main lobe of the tag facing the reader antenna. The tags were attached to a piece of EPS (styrofoam) to keep them upright. The measurements were made in the frequency range from 800 to $1000 \mathrm{MHz}$. The measurement distance was $45 \mathrm{~cm}$, and the highest transmitter EIRP power was $3.28 \mathrm{~W}$, as permitted in Europe.

\section{Results and Discussion}

In this section, we will begin by addressing the effect of the substrate material. The antennas are first measured right after coating the IC, then soaked and laundered. The effect of moisture and mechanical stress in laundering on each coating material is analyzed.

5.1. Effect of Substrate Material. Generally, the substrate has an effect on the tag performance through its electrical properties such as loss tangent and relative permittivity. Loss tangent affects the radiation efficiency of the tag antenna, and 


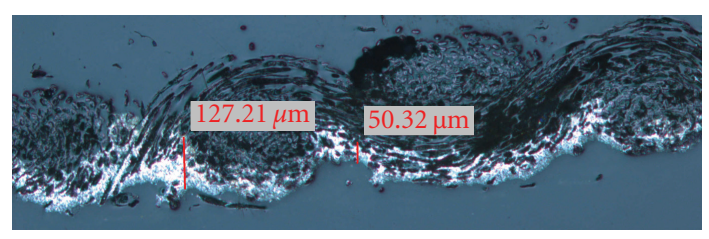

(a)

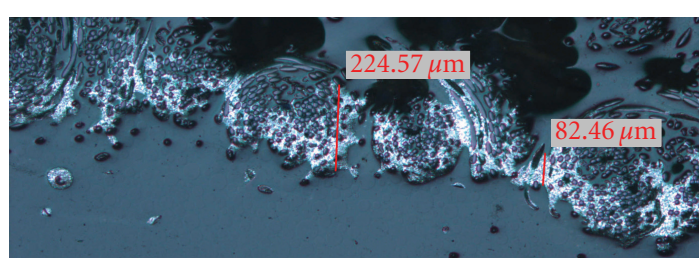

(b)

Figure 3: Cross-sections of antennas on Fabric (A) and Fabric (B), magnification is $\times 20$. The light part in the figure is the ink film, and the dark part is the fibre structure of the fabric.

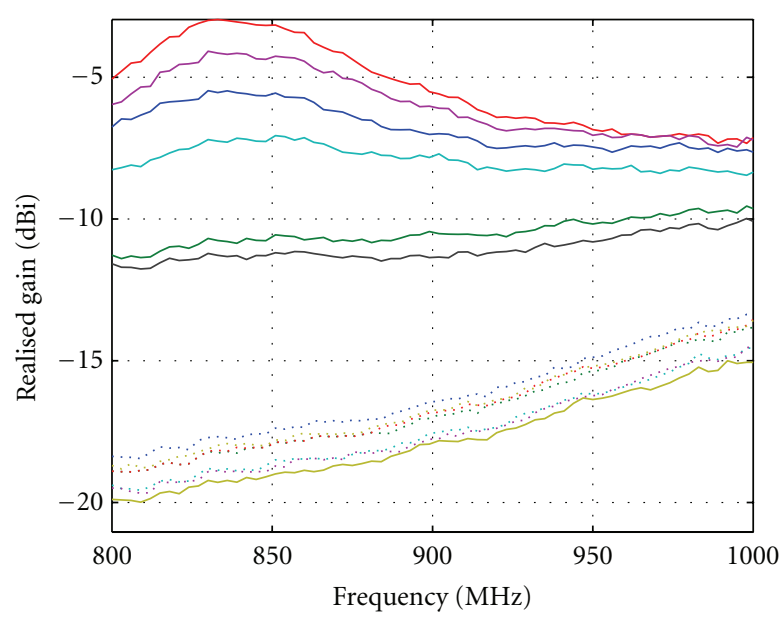

\begin{tabular}{|c|c|}
\hline A, no coat & … B, no coat \\
\hline A, epoxy & B, epoxy \\
\hline A, silicone & $\mathrm{B}$, silicone \\
\hline A, latex & B, latex \\
\hline A, PVA1 & B, PVA1 \\
\hline A, PVA2 & B, PVA2 \\
\hline
\end{tabular}

(a)

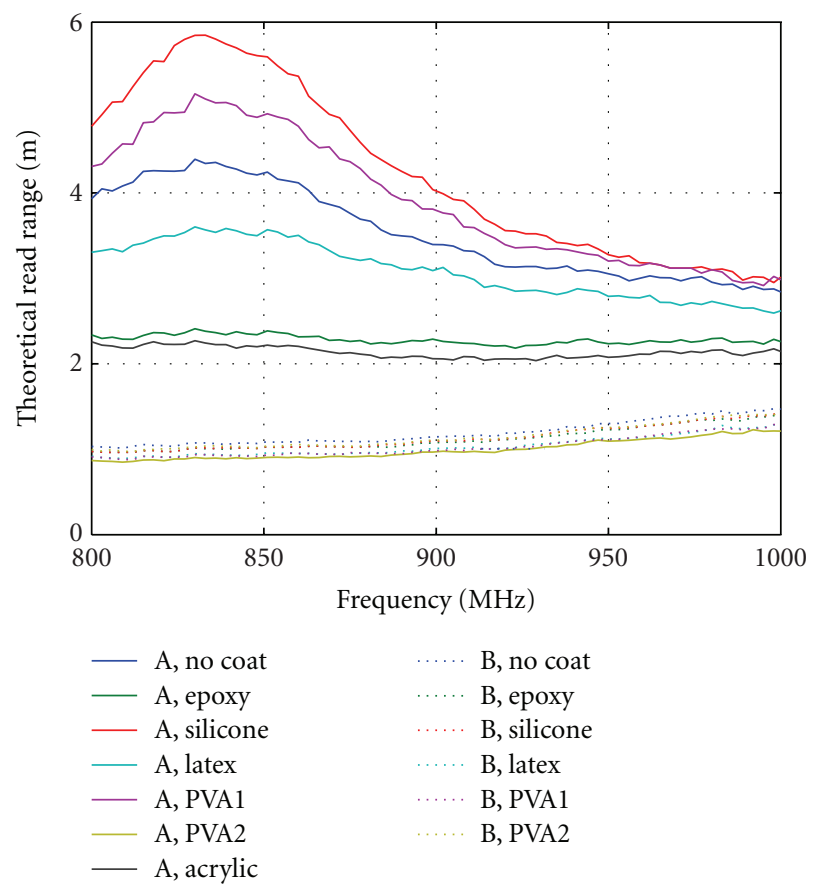

(b)

FIGURE 4: Frequency responses of the dry tags, expressed as realised gain as well as theoretical read range. All the tags on fabric (B) operate poorly because of the broken $t$-matching section.

relative permittivity mainly affects the operation frequency. Porous substrates also have an indirect effect on the tag through the ink film morphology: it depends on the printing method, substrate, and composition of the ink. On porous substrates, the ink is partially absorbed into the substrate, and the average thickness of the printed film depends on the substrate material. Thickness variation occurs also on smooth nonabsorbent substrates, but the variation is most obvious on porous and rough substrates such as fabrics. The cross section area of the conductive film is an important parameter that affects the electrical performance of a film printed with conductive inks. Both the surface profile and the thickness of the film have an effect on the ink film cross section area, and thus the substrate affects the ink film electrical performance $[21,24,27]$.

The threshold powers of all the fabricated tags were measured after coating the IC. There was a notable difference between the two fabrics: tags printed on Fabric (B) (knitted) performed poorly, as seen in Figure 4. This is explained through the surface structure: the surface of Fabric (B) is more uneven compared to Fabric (A), due to the interlocking loops of the knitted fabric. Textured or irregular surfaces are complicated because there are many levels to print. It is easy to print on the raised areas but the transfer of the ink onto recessed areas is difficult, leading to an uneven ink film. This becomes particularly significant in narrow conductive traces of the printed pattern. In such places, the ink film may be almost broken depending on the position of the loops and the trace. In addition, the ink is partially absorbed into the fabric but not inside the fibres of the fabric. Our geometry includes a narrow trace in the $\mathrm{t}$-match structure (width $0.5 \mathrm{~mm}$ ). Since the $\mathrm{t}$-match plays an important role in impedance matching to the IC, poor print quality, particularly on Fabric (B) (uneven and too narrow trace, as seen 


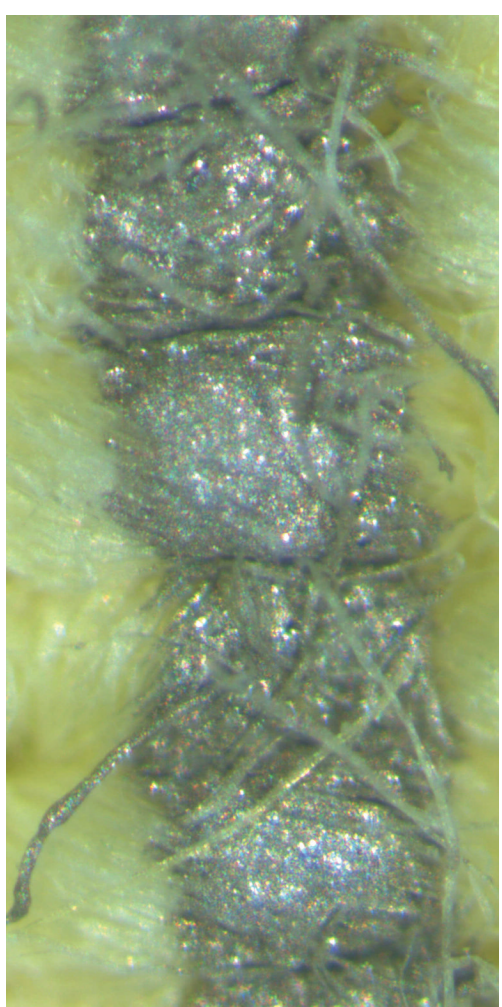

(a)

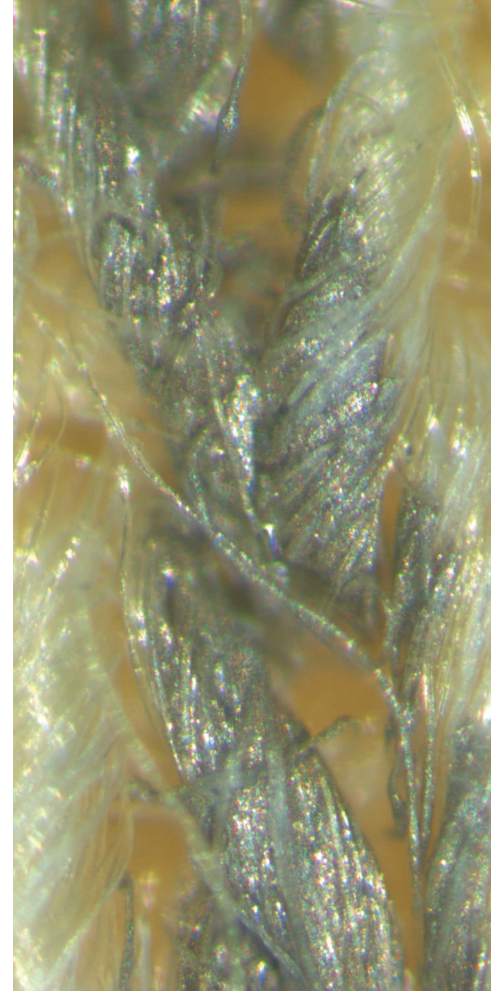

(b)

FIgURE 5: T-match structure on Fabric (A) and Fabric (B), magnification is $\times 57$.

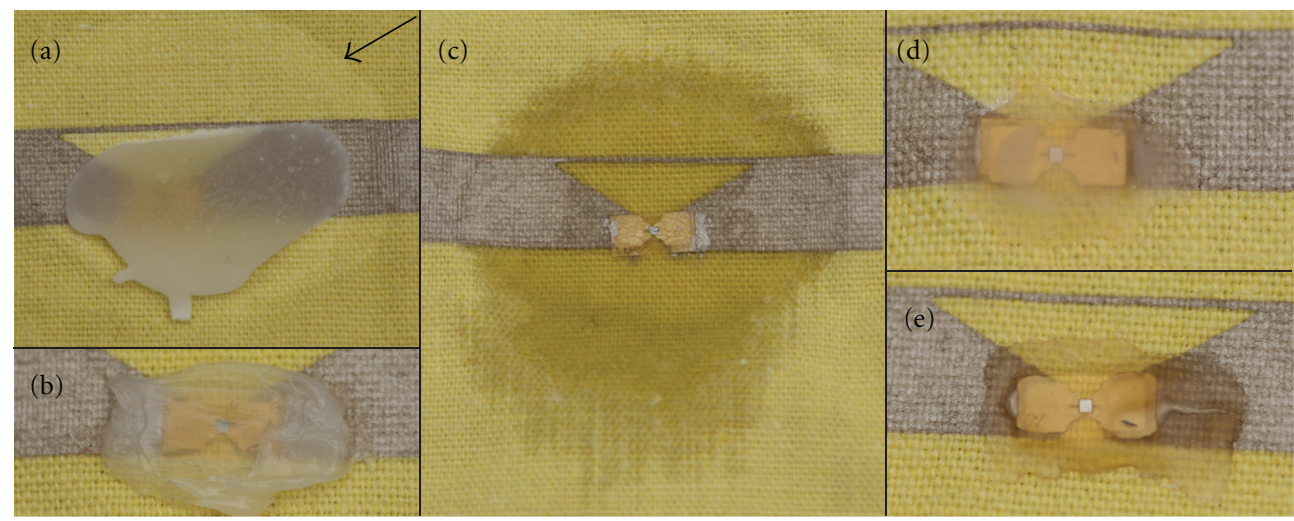

Figure 6: Photographs of the coated ICs on Fabric (A). (a) Acrylic; larger acrylic lump on the backside is visible as a white fabric, indicated with an arrow. This hard piece of acrylic makes the t-matching section hard, which possibly prevented it from breaking in laundry. (b) Silicone. (c) Epoxy. (d) PVA2 glue (PVA1 glue looks similar). (e) Latex glue.

in Figure 5), is supposed to affect the tag antenna behaviour essentially. In [28], poor print quality in the t-match was affecting gravure-printed tag on paper in the same way.

Possible solutions to the problem would be to print thicker ink films, to use a primer to smoothen the ink film, to select a denser fabric substrate, or to use tag geometry with wider conductors. Fabric (A) is denser and smoother than Fabric (B), and most of the tags printed on Fabric (A) had an intact t-matching section. However, mechanical stress especially in laundering was later seen to damage the tags printed on Fabric (A) as well.
5.2. Antennas with Coated IC. Table 3 summarises the dimensions and findings of the different coatings. Photographs of the coated ICs are shown in Figure 6.

When epoxy was applied on the tag, it was absorbed into the textile in an uncontrollable fashion in an area of $2 \mathrm{~cm}$ in diameter. This area becomes hard and therefore potentially uncomfortable. Moreover, when the epoxy covers the $t$ matching section (the thin line), it can alter the inductance of the section and thus the antenna impedance. Possibly, if the epoxy is allowed to harden for a while before it is applied on the tag, spreading could be better controlled. Both the 


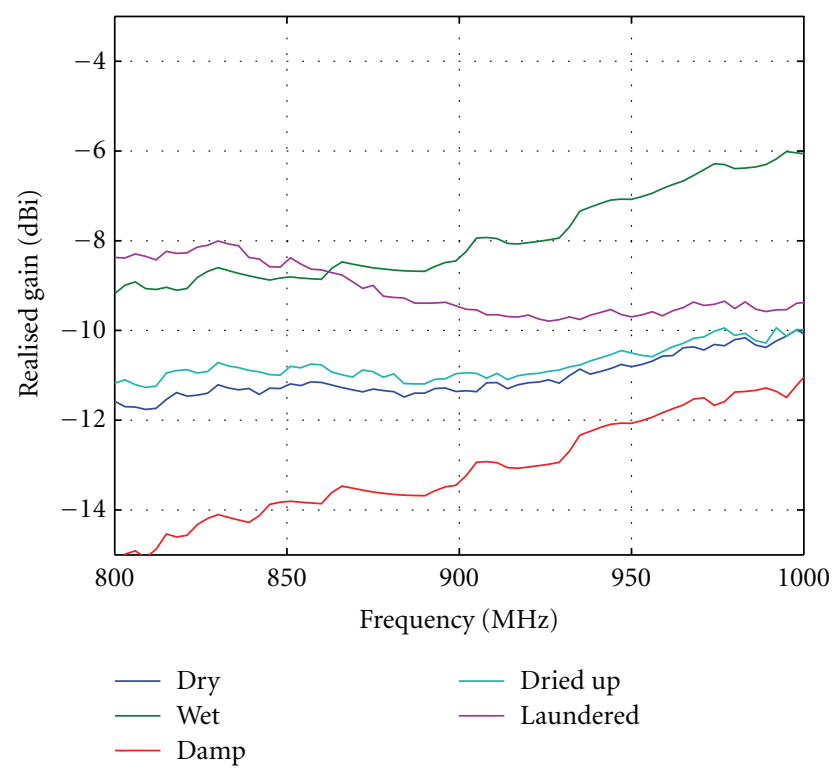

(a)

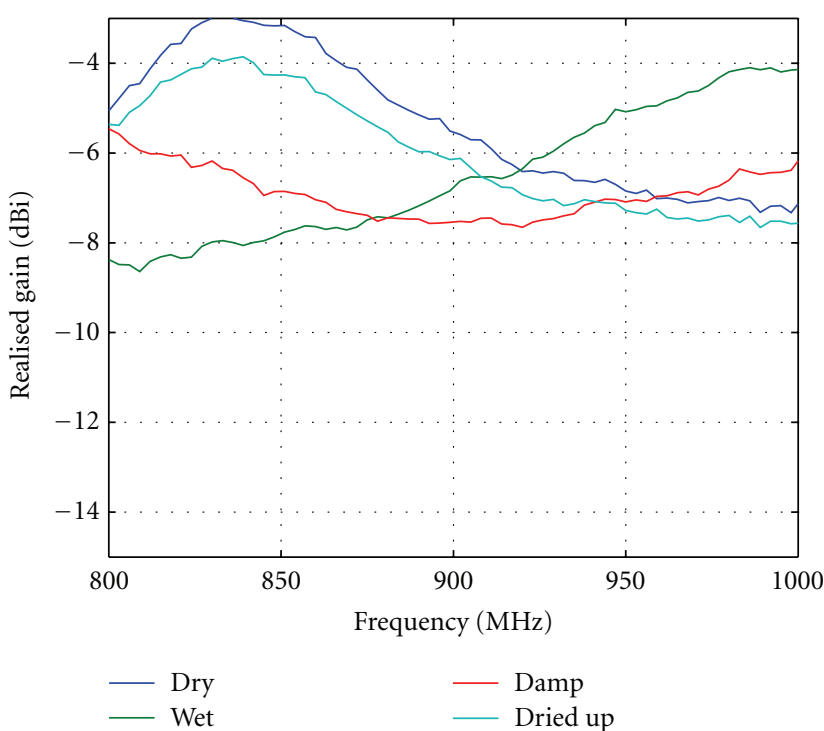

(b)

FIGURE 7: Realised gains of the tags measured before and after soaking, damp, dried up again, and after laundering (if available). (a) Acrylic coated tag; (b) silicone coated tag; both on Fabric (A).

TABLE 3: Observations and thicknesses of the tags with a coated IC. Fabric A is approximately $0.4 \mathrm{~mm}$ thick and Fabric B $0.8 \mathrm{~mm}$ thick. The IC strap is $0.3 \mathrm{~mm}$ thick.

\begin{tabular}{lcc}
\hline Coating & $\begin{array}{c}\text { Thickness } \\
\text { (two layers + fabric) }\end{array}$ & Notes \\
\hline Epoxy & 0.5 to $1 \mathrm{~mm}$ & $\begin{array}{c}\text { Is absorbed and spreads; all the } \\
\text { measured tags have broken } \\
\text { t-match }\end{array}$ \\
Acrylic & 5 to $8 \mathrm{~mm}$ & Hard lump \\
Silicone & 2 to $3 \mathrm{~mm}$ & \\
Latex & 1.5 to $2 \mathrm{~mm}$ & \\
PVA1 & 1.5 to $2 \mathrm{~mm}$ & \\
PVA2 & 1.5 to $2 \mathrm{~mm}$ & \\
\hline
\end{tabular}

epoxy coated tags on Fabric (A) (two samples) had a broken t-match, which could be due to the liquid epoxy filling the microcracks in the conductor and thus open-circuiting it. More samples are needed to test this hypothesis.

Acrylic was hardly absorbed in the fabric at all, and because it hardens immediately, it remained on the tag as a hard lump, although well adhered to the fabric. The total thickness of the acrylic coated IC was 5 to $8 \mathrm{~mm}$. It was difficult to prevent the acrylic from covering the t-match section.

The glue-type coatings (PVA1, PVA2, latex, silicone) were easily applied to the textile so that only the area containing the IC strap was covered. After drying, the PVA1 and PVA2 glues became hard, while silicone and latex stayed flexible.

All the tags were operational immediately after the coating was applied. In Figure 4, we can see two groups of frequency responses: tags with broken and intact $\mathrm{t}$-matches.
TABLE 4: Findings of the moisture test.

\begin{tabular}{lcc}
\hline Coating & $\begin{array}{c}\text { Effect of soaking cycle } \\
\text { on realised gain }\end{array}$ & Notes \\
\hline Epoxy & N/A & $\begin{array}{c}\text { No data, all the tags had broken } \\
\text { t-match }\end{array}$ \\
Acrylic & $-0.5 \mathrm{~dB}$ & \\
Silicone & $+1 \mathrm{~dB}$ & \\
Latex & $-2 \mathrm{~dB}$ & $\begin{array}{c}\text { Dissolves in water, rejected } \\
\text { PVA1 }\end{array}$ \\
PVA2 & $-1 \mathrm{~dB}$ & $\begin{array}{c}\text { Partially dissolves, potentially } \\
\text { could wear out in repeated } \\
\text { washing }\end{array}$ \\
\hline
\end{tabular}

The silicone coated tag on Fabric (A) performs the best at this point. After the first step, coating the ICs of the tags, we rejected none of the coatings. However, glue-type coatings were seen easier to handle than epoxy and acrylic. It is though worth remembering that in the industry the coatings are automatically applied by dispensers, for example. This makes the handling of the coatings easier but does not prevent absorption and spreading of the coatings (such as epoxy) in the fabric.

5.3. Soaking Test. The tags performed equally before and after soaking and complete drying. Only minor differences of the order of $3 \mathrm{~dB}$ or less were observed in the realised gain. Figure 7 illustrates how the response returns to the original state. The frequency response of the damp tags (water content 10\%) was between the dry and the wet cases, an exception being the damp acrylic coated tag shown in Figure 7. 


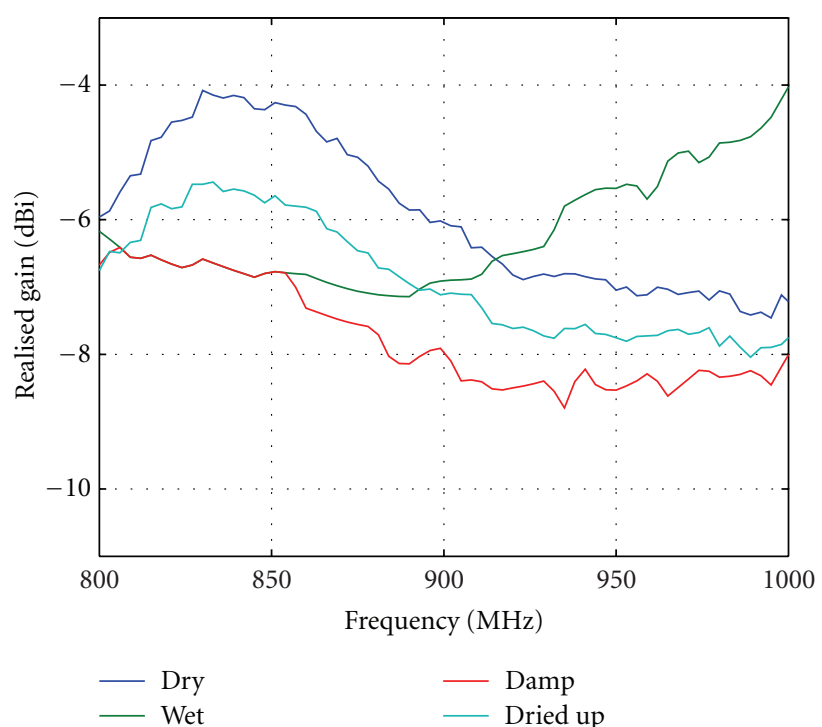

(a)

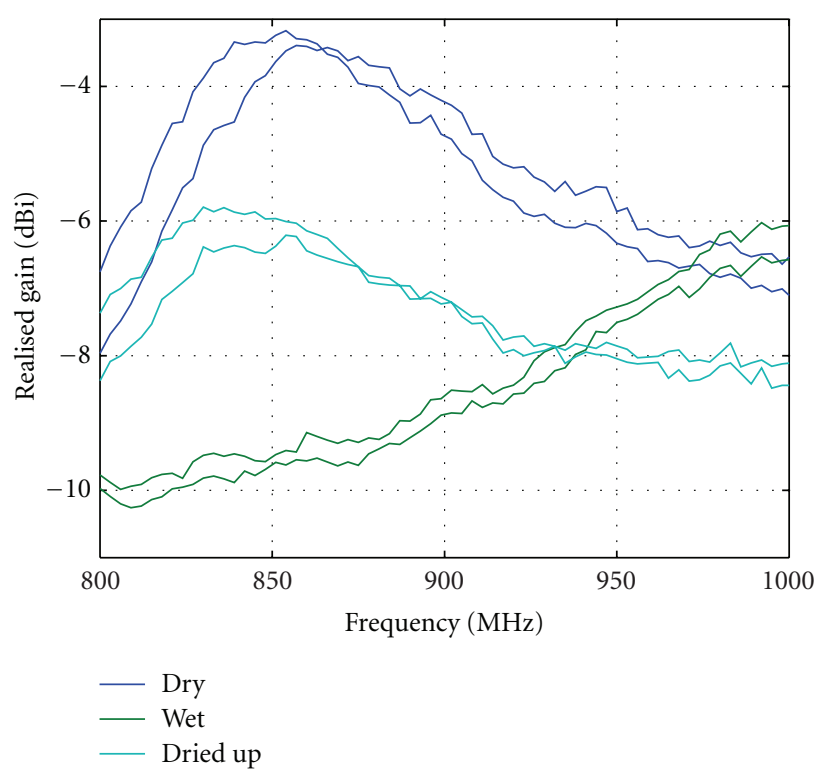

(b)

FIgURE 8: (a) PVA1 glue coated tag; (b) PVA2 coated tag (two samples); all on Fabric (A). Even though the PVA1 glue dissolves in water, the tag coated with this wet glue performs well. After drying up, the glue and the PVA1 coated tag operate like before washing, with a $1.5 \mathrm{~dB}$ decrease in realised gain. The gain of the PVA2 coated tag decreases by $3 \mathrm{~dB}$.

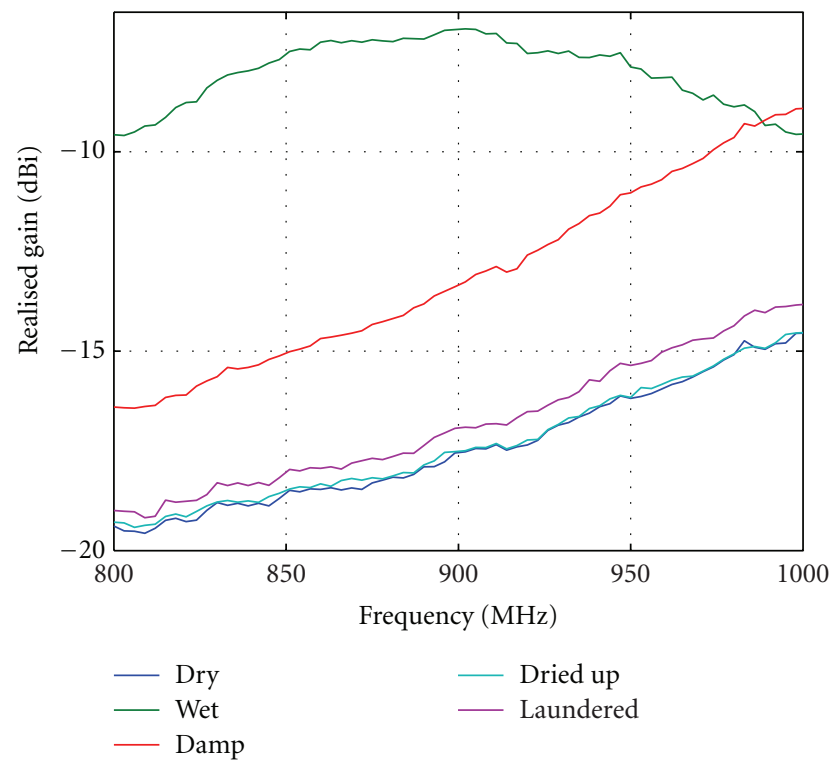

FIGURE 9: Frequency response of a tag with a broken t-match (Fabric (B), latex coating). When the tag is soaked, the matching section becomes conductive and the tag operates normally.

The PVA1 glue is not water resistant. Even when the dry glue is sprayed with water, it starts to dissolve and becomes white. Still, the tag was operational although the IC coating was wet. After drying up again, the glue looked intact and the tag performance was almost the same as before soaking, as seen in Figure 8. However, if the glue undergoes any mechanical stress (such as rubbing with fingers) when it is moist, it easily comes off. Therefore, this coating was rejected.
When the waterproof PVA2 glue was soaked, some colour change was observed on the edges. The major part of the glue was still intact, but we believe that repeated washing cycles would gradually dissolve the glue. It is therefore questionable whether this glue can be used to protect the IC. The measured realised gain of the PVA2 coated tag deteriorated $3 \mathrm{~dB}$ in one soaking cycle.

When tags with a broken $\mathrm{t}$-matching section were soaked, the operation of the tag improved to nominal, as seen in Figure 9. It appears that in a wet tag water droplets enter the microcracks in the $t$-matching section, restoring the conductivity. Thus the wet tag operates well, but when the tag is allowed to dry, the operation becomes poor again. However, more tests are needed to confirm this.

Table 4 summarises the findings of the moisture test. After the moisture test step, we must reject the PVA1 coating.

5.4. Laundry Test with One Wash. The frequency responses of the tags were measured after one laundry cycle. As expected, the nonwaterproof PVA1 glue came off completely with washing. Visual inspection showed no significant changes in the other coating materials. The IC was not detached from any of the samples (except PVA1), which shows that even flexible coating materials can be used to protect the IC from mechanical stress.

Machine washing with spinning caused major wear to the screen-printed conductor. The colour of the conductor changed from silvery to darker grey, as seen in Figure 10, due to chemical reactions such as oxidation of the silver, which also occurs when a tag is kept in touch with air for several months. The silver is in small particles in the ink solution, which is why there is plenty of reactive silver surface on the printed film surface to react with air, water, detergent, and so 


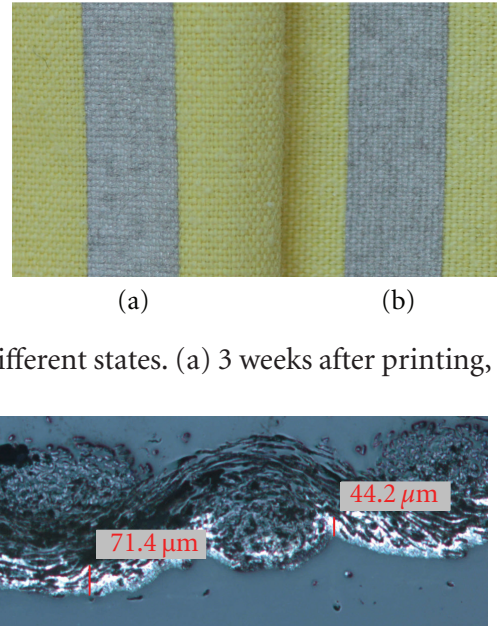

(a)

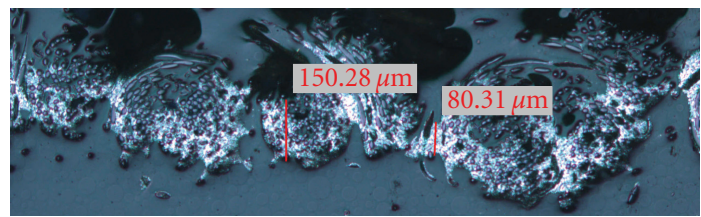

(b)

Figure 11: Cross-sections of antennas on Fabric (A) and Fabric (B), washed ten times, magnification is $\times 20$.

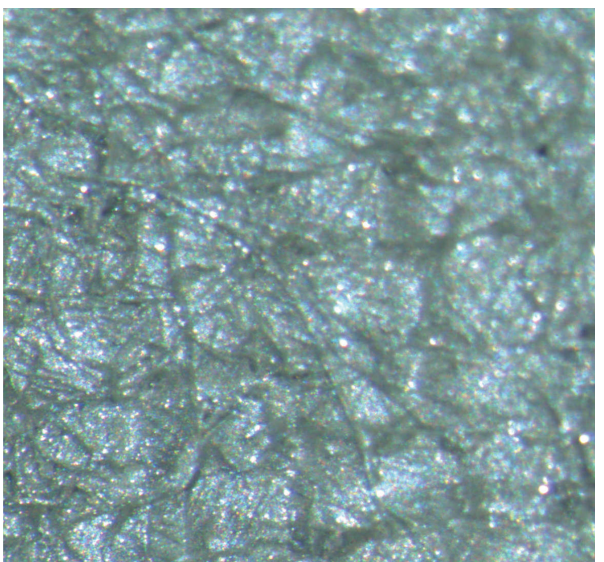

(a)

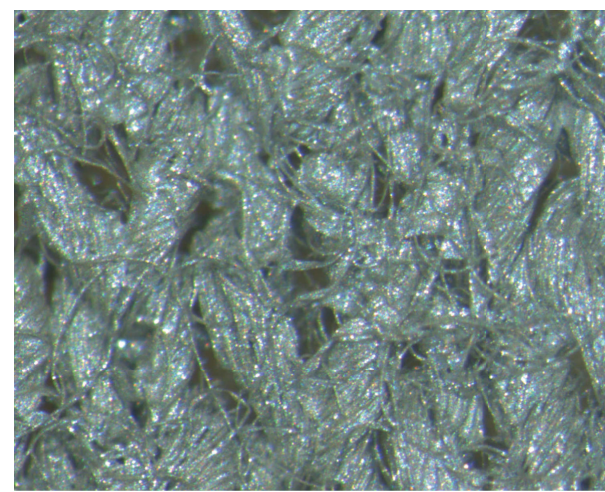

(c)

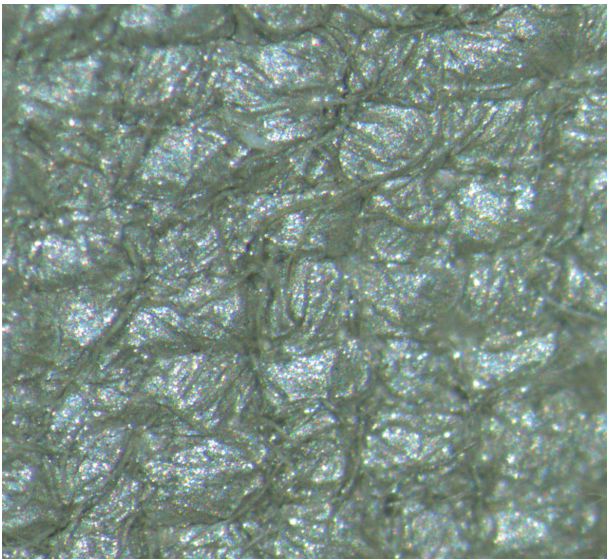

(b)

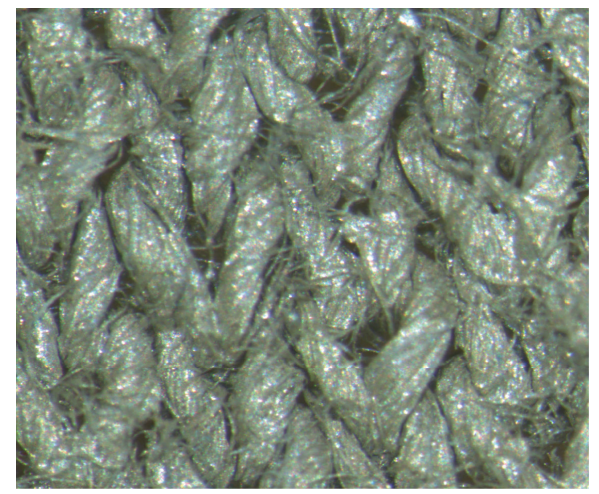

(d)

FIGURE 12: Antenna surface on Fabric (A): (a) before washing and (b) after ten washes. Antenna surface on Fabric (B): (c) before washing and (d) after ten washes. Magnification is $\times 20$.

forth. However, the oxides of silver are electrically conductive and do not cause dramatic changes to the ink film. Further work is needed to investigate what other compounds are found in the laundered ink film surface. After laundry, the $\mathrm{t}$-matching section was broken in all but one of the tags. This calls for extra protection of the narrow conductor in addition to the IC coating.

Acrylic coating seemed to protect the thin t-match in addition to the IC, because the hard piece of acrylic extended over the $\mathrm{t}$-matching section. It is not known whether flexible 
coatings over the t-matching section would provide similar protection, but this is a topic for future research.

5.5. Antenna Laundry Test with Ten Washes. Cross-sections of antennas on Fabrics (A) and (B) after ten washing cycles can be seen in Figure 11. The tag surface on Fabric (A) and Fabric (B) before washing and after ten washes can be seen in Figure 12. According to these figures, no changes in the antennas can be seen after ten washings, apart from the colour change. However, during washing the tags go through strong mechanical stresses which may cause micro-cracks on the antenna surface and also affect the IC joint. Thus, mechanical stresses may have an effect on the tag performance even though clear changes cannot be seen.

In [6], washing tests such as dry cleaning and waterwashing with the detergent were performed to screenprinted circuits on fabrics. The samples without passivation material coating experienced thinning of wire thickness and also open connections. Their resistance values increased to 40 times of the original value after about 20 washes. With the passivation material, their endurance against washing increased (resistance twice the original value). This encourages us to further investigate the possibilities of different coating materials also in case of RFID tags.

\section{Conclusion}

We have investigated the performance of a wearable RFID tag screen-printed on a fabric substrate. Two fabrics and six coating materials were tested.

The print quality plays a very important role especially in thin conductive traces in the RFID tag geometry. Geometries that contain narrow traces may limit the substrate material choice. It was seen that the flexible and relatively loose, interlock knitted Fabric (B) was not suitable for the tag geometry in question. The tag includes a thin matching section with dimensions $0.5 \mathrm{~mm}$ by $17 \mathrm{~mm}$. The uneven fabric surface ( 1 stitch $\approx 0.8 \mathrm{~mm}$ ) caused discontinuities and breaks in the thin conductor, making the tag very poorly matched to the IC and hence reducing the realised gain and read range. This is in accordance with the results published in [6]: the denser and smoother the fabric, the better. Ways to protect thin conductor lines should be investigated, along with the minimum reasonable line width.

In the first tests, only one of the coating materials was rejected right away, because it was not water resistant. Silicone and latex remain flexible even after drying, whereas epoxy, PVA2, and acrylic become hard. However, hard coatings may be needed to keep thin conductors from breaking in laundry. Generally, glue-type coating materials were easier to handle and could be spread precisely. Although laundering presents a major mechanical stress to the IC, any coating material was sufficient to protect it from breaking off.

More reliability testing work is needed in order to examine the potential of these RFID tags in normal field use, as well as the effect of washing and drying cycles on the tags. Possible tests are, for example, bending tests, high temperature tests, and high temperature high pressure steam tests (industrial laundry). Environmental testing of electrically conductive adhesives in the IC strap joint will help to make the contact more reliable. Also, normal use of clothing with body-worn RFID tags needs to be tested, and laundry tests must be repeated with more washing cycles.

\section{Acknowledgments}

This work was funded by the Finnish Cultural Foundation Pirkanmaa Regional Fund, Helsingin Sanomat Foundation, the European Social Fund (ESF), and the Centennial Foundation of Finnish Technology Industries (Teknologiateollisuuden 100-vuotissäätiö).

\section{References}

[1] "IDS Microchip AG. SL900A, EPC class 3 sensory tag chip," September 7, 2012, http://ids-microchip.com/s1900a.php.

[2] M. Prudenziati, Ed., Thick Film Sensors, Elsevier, 1994.

[3] W. T. Hicks, T. R. Allington, and V. Johnson, "Membrane touch switches: thick film materials systems and processing options," IEEE transactions on components, Hybrids, and Manufacturing Technology, vol. 3, no. 4, pp. 518-524, 1980.

[4] Alien Technology, "Higgs-2 EPC Class 1 Gen 2 RFID Tag IC, Product overview," July 23, 2012, http://www.alientechnology .com/docs/products/DS_H2.pdf.

[5] T. Kellomäki and L. Ukkonen, "Design approaches for bodyworn RFID Tags," in Proceedings of the 3rd International Symposium on Applied Sciences in Biomedical and Communication Technologies (ISABEL '10), Rome, Italy, November 2010.

[6] Y. Kim, H. Kim, and H.-J. Yoo, "Electrical characterization of screen-printed circuits on the fabric," IEEE Transactions on Advanced Packaging, vol. 33, no. 1, pp. 196-205, 2010.

[7] J. Heikkinen, T. Laine-Ma, A. Ruhanen, and M. Kivikoski, "Flexible antennas for GPS reception," in Proceedings of the European Conference on Antennas and Propagation (EuCAP '06), November 2006.

[8] S. Merilampi, T. Björninen, L. Ukkonen, P. Ruuskanen, and L. Sydänheimo, "Embedded wireless strain sensors based on printed RFID tag," Sensor Review, vol. 31, no. 1, pp. 32-40, 2011.

[9] S. Merilampi, T. Björninen, L. Ukkonen, P. Ruuskanen, and L. Sydänheimo, "Printed passive UHF RFID tags as wearable strain sensors," in Proceedings of the 3rd International Symposium on Applied Sciences in Biomedical and Communication Technologies (ISABEL '10), Rome, Italy, November 2010.

[10] L. Vallozzi, H. Rogier, and C. Hertleer, "Dual polarized textile patch antenna for integration into protective garments," IEEE Antennas and Wireless Propagation Letters, vol. 7, pp. 440-443, 2008.

[11] S. Zhu and R. Langley, "Dual-band wearable textile antenna on an EBG substrate," IEEE Transactions on Antennas and Propagation, vol. 57, no. 4, pp. 926-935, 2009.

[12] J. C. G. Matthews and G. Pettitt, "Development of flexible, wearable antennas," in Proceedings of the 3rd European Conference on Antennas and Propagation (EuCAP '09), pp. 273-277, March 2009.

[13] T. Maleszka, M. Preisner, and P. Kabacik, "Meshed ground plane structures for textile antennas," in Proceedings of the $3 \mathrm{rd}$ European Conference on Antennas and Propagation (EuCAP '09), pp. 713-717, March 2009.

[14] J. S. Roh, Y. S. Chi, J. H. Lee, Y. Tak, S. Nam, and T. J. Kang, "Embroidered wearable multiresonant folded dipole antenna 
for FM reception," IEEE Antennas and Wireless Propagation Letters, vol. 9, pp. 803-806, 2010.

[15] S. Park, K. Mackenzie, and S. Jayaraman, "The wearable motherboard: a framework for personalized mobile information processing (PMIP)," in Proceedings of the 39th Annual Design Automation Conference, pp. 170-174, June 2002.

[16] E. R. Post, M. Orth, R. R. Russo, and N. Gershenfeld, "Ebroîdery: design and fabrication of textile-based computing," IBM Systems Journal, vol. 39, no. 3-4, pp. 840-860, 2000.

[17] J. Edmison, M. Jones, Z. Nakad, and T. Martin, "Using piezoelectric materials for wearable electronic textile," in Proceedings of the International Symposium on Wearable Computers, pp. 41-48, October 2002.

[18] D. Cottet, J. Grzyb, T. Kirstein, and G. Tröster, "Electrical characterization of textile transmission lines," IEEE Transactions on Advanced Packaging, vol. 26, no. 2, pp. 182-190, 2003.

[19] S. Jung, C. Lauterbach, and W. Weber, "A digital music player tailored for smart textiles: first results," in Proceedings of the International Avantex Symposium, p. 15, 2002.

[20] J. J. Heikkinen, T. T. Laine-Ma, and M. A. Kivikoski, "Flexible fabric-base patch antenna with protective coating," in Proceedings of the IEEE Antennas and Propagation Society International Symposium, AP-S, pp. 4168-4171, June 2007.

[21] S. L. Merilampi, T. Bjorninen, A. Vuorimaki, L. Ukkonen, P. Ruuskanen, and L. Sydanheimo, "The effect of conductive ink layer thickness on the functioning of printed UHF RFID antennas," Proceedings of the IEEE, vol. 98, no. 9, pp. 16101619, 2010.

[22] H. Kipphan, Handbook of Print Media, Springer, 2001.

[23] S. Merilampi, T. Laine-Ma, and P. Ruuskanen, "The characterization of electrically conductive silver ink patterns on flexible substrates," Microelectronics Reliability, vol. 49, no. 7, pp. 782790, 2009.

[24] S. B. Hoff, Screen Printing-A Contemporary Approach, Delmar Publishers, 1997.

[25] H. Ujiie, Digital Printing of Textiles, Woodhead Publishing, 2006.

[26] "Voyantic Ltd.," August 8, 2012, http://voyantic.com.

[27] S. L. Merilampi, T. Björninen, L. Ukkonen, P. Ruuskanen, and L. Sydänheimo, "Characterization of UHF RFID tags fabricated directly on convex surfaces by pad printing," The International Journal of Advanced Manufacturing Technology, vol. 53, no. 5-8, pp. 577-591, 2011.

[28] T. Björninen, S. Merilampi, L. Ukkonen, L. Sydänheimo, and P. Ruuskanen, "The effect of fabrication method on passive UHF RFID tag performance," International Journal of Antennas and Propagation, vol. 2009, Article ID 920947, 8 pages, 2009.

[29] "Buehler Varikleer product info," August 8, 2012, http://www .buehler.co.uk/fileadmin/user_upload/pdf/productinfo/VariKleer.pdf.

[30] "Struers cold mounting brochure," August 8, 2012, http:// www.struers.com/resources/elements/12/147271/Cold_Mounting_brochure_English.pdf.

[31] "Bison textile adhesive datasheet," August 8, 2012, http:// www.bison.net/static/products/assets/asset_8364_3.pdf.

[32] "Casco silicone datasheet," August 8, 2012, http://www.casco .fi/_upl/pdf/2992\%20Casco\%20silikoni.pdf.

[33] "Erikeeper webpage," August 8, 2012, http://www.erikeeper.fi. 

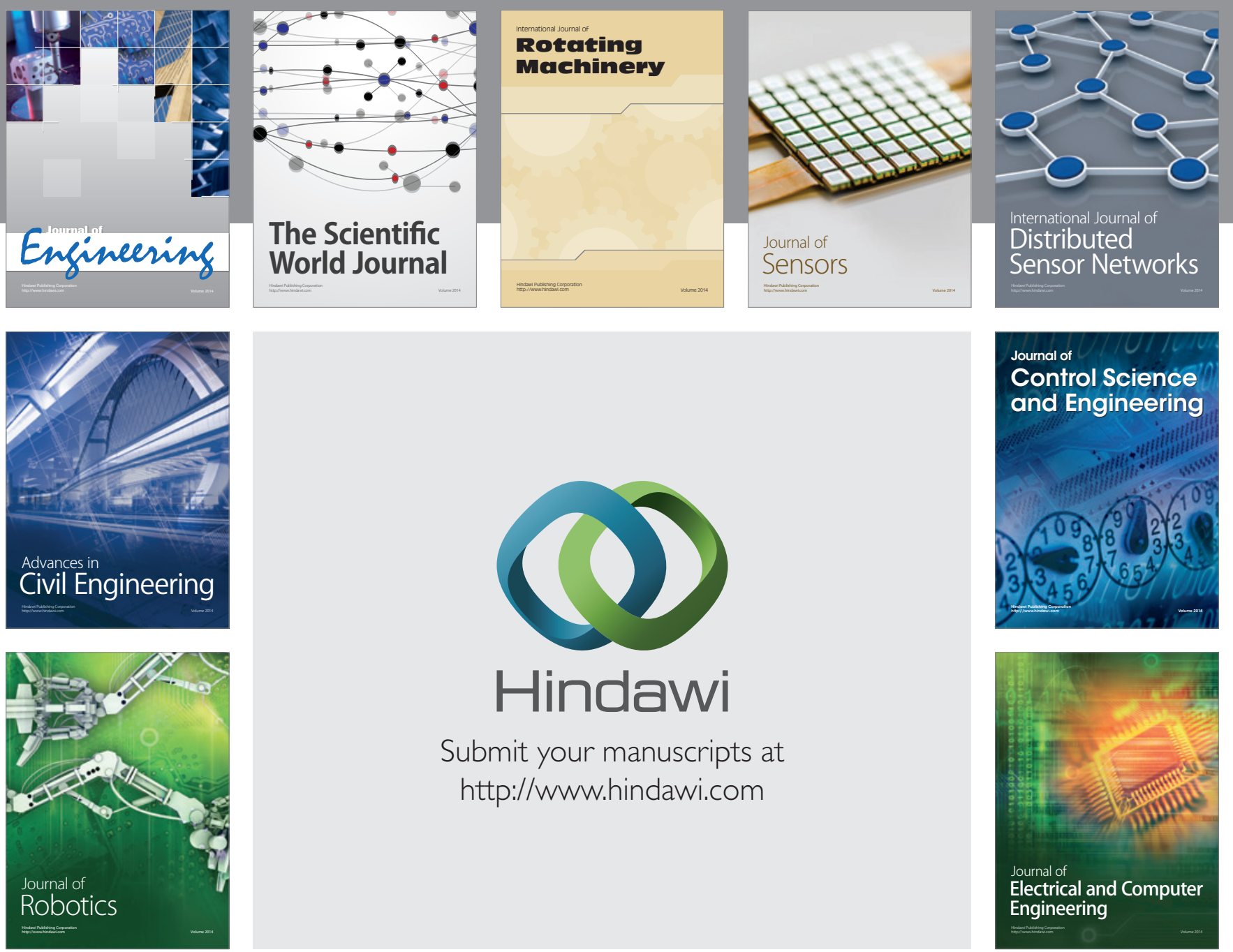

Submit your manuscripts at

http://www.hindawi.com
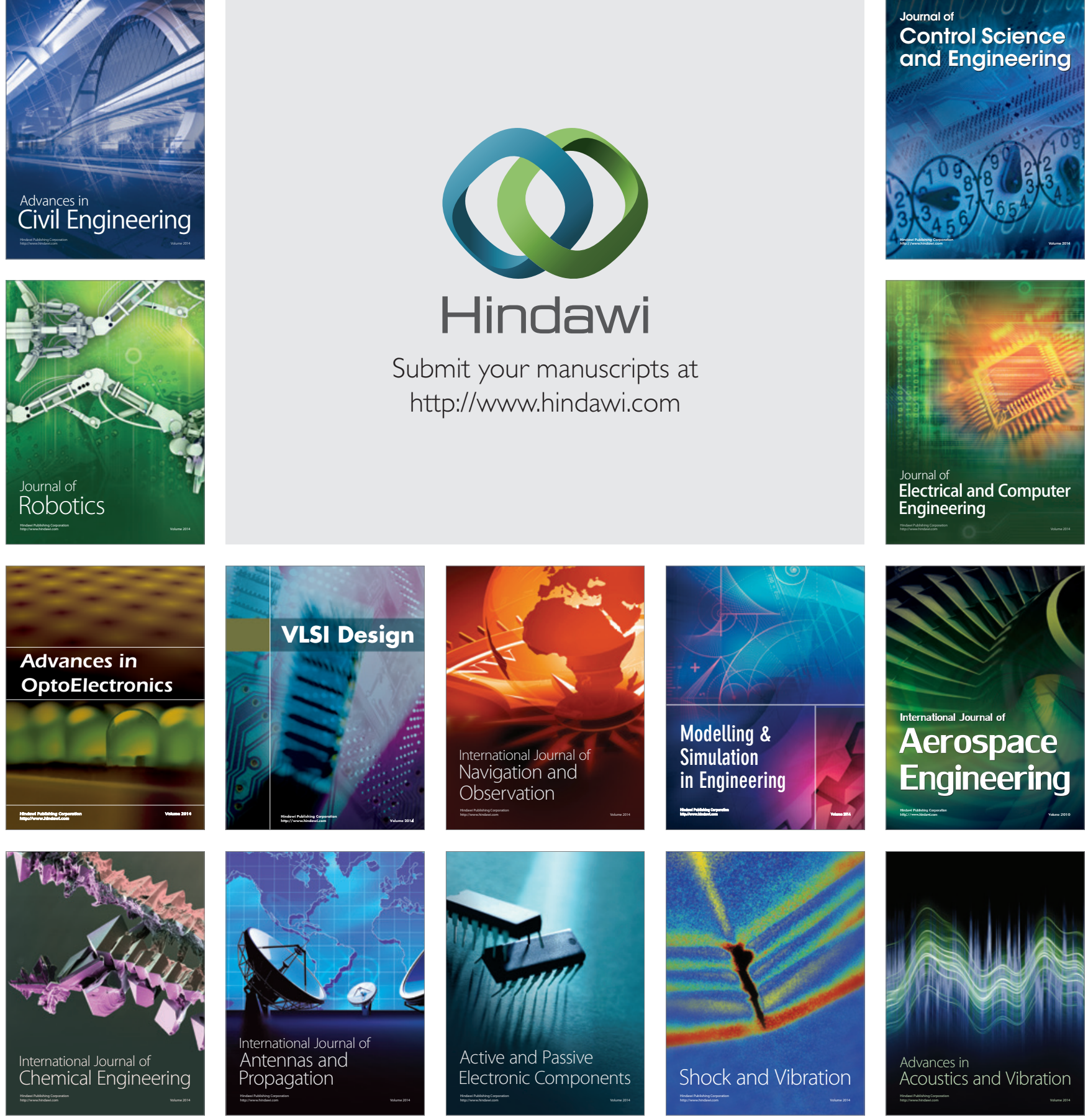